\title{
Closing the door on KRAS-mutant lung cancer
}

Despite considerable effort to target KRAS mutations, which are particularly common in pancreatic, colorectal and lung cancers, personalized therapies are not clinically available, and these cancers remain among the most lethal. A screen in non-small-cell lung cancer (NSCLC) cells has identified nuclear export proteins, particularly the nuclear export receptor exportin 1 (XPO1), as crucial to mutantKRAS-driven cancer; disruption of this export machinery in cell and mouse models can prevent growth of tumours harbouring KRAS mutations.

First, the authors clustered 106 NSCLC cell lines according to whole-genome mRNA expression

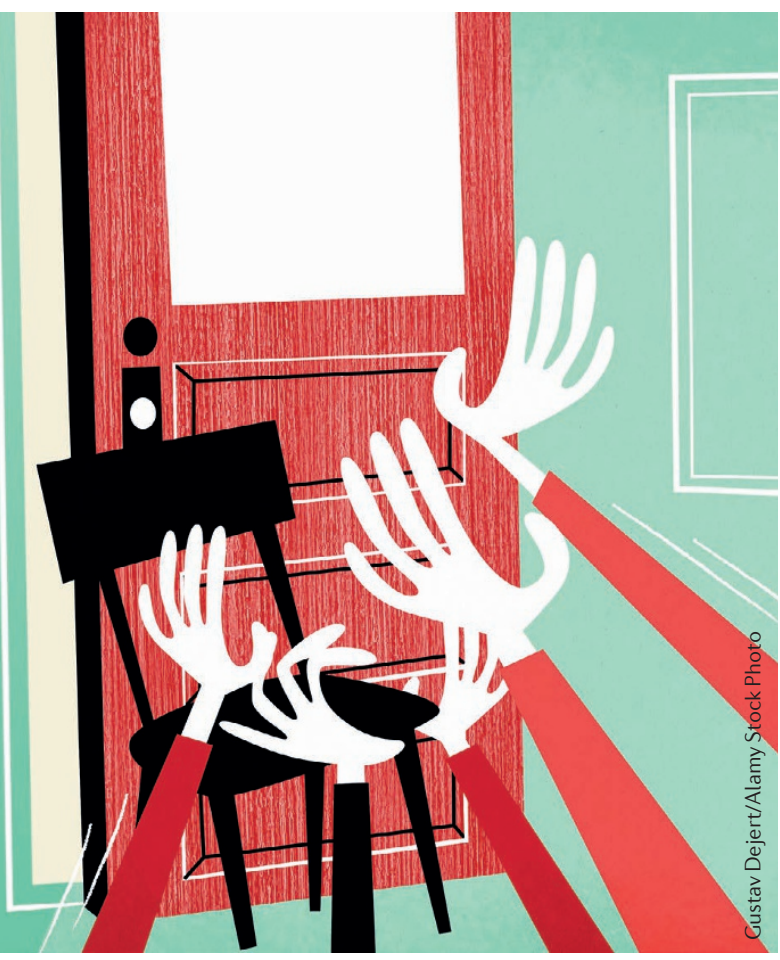

patterns. At least eight phenotypic clusters emerged, and mutant KRAS

alterations in the subcellular localization of certain

proteins, particularly $\mathrm{I} \kappa \mathrm{B} \alpha$ and YAP, could contribute to KRAS-mutant cancer growth

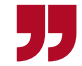
specifically with mutant KRAS (and not other factors associated with including 6 with mutant KRAS, with and performed a genome-wide siRNA synthetic lethality screen. They identified ten gene sets whose inhibition selectively killed NSCLC cell lines with mutant $K R A S$, all of which contained genes coding for nuclear transport machinery.

$\mathrm{XPO} 1$ is a druggable component lines were present in most of them. To search for synthetic lethal interactions clustering), they selected 12 cell lines, a range of mRNA expression patterns of nuclear export, and the orally available XPO1 inhibitor KPT-330 is currently in phase II trials for haematological and solid tumours. Pharmacological inhibition of XPO1 selectively induced apoptosis in cell lines with mutant KRAS. Similarly, in xenograft, patient-derived xenograft or genetically engineered mouse models of KRAS-mutant NSCLC, treatment with KPT-330 prevented tumour growth.

KRAS can activate nuclear factor $-\kappa B(N F-\kappa B)$, and expression of NF- $\kappa \mathrm{B}$ target genes was elevated in the XPO1 inhibitor-sensitive cell lines. In cells treated with XPO1 inhibitors, NF- $\kappa \mathrm{B}$ inhibitor- $\alpha(\mathrm{I} \kappa \mathrm{B} \alpha)$ accumulated in the nucleus, where it inhibits NF- $\mathrm{BB}$-dependent transcription. Cells carrying a drug-resistant version of XPO1 did not undergo apoptosis, and NF- $\kappa \mathrm{B}$ activity was not perturbed by drug treatment. Similarly, siRNA targeting I $\kappa \mathrm{B} \alpha$ and the related I $\mathrm{B} \beta \beta$ was sufficient to render cells resistant to XPO1 inhibitors, and overexpression of a form of I $\mathrm{B} a$ that lacked a nuclear export signal killed KRAS-mutant, but not wildtype, NSCLC cells. Although at least one form of I $\kappa \mathrm{B}$ accumulated in the nuclei of all cells treated with XPO1 inhibitors, this was associated with apoptosis only in those with mutant KRAS, suggesting the potential for cancer-cell-specific drug targeting.

A second mechanism could also be at play. KRAS-mutant, XPO1 inhibitor-resistant cell lines contained mutations in the mechanistically uncharacterized gene follistatin-like 5 (FSTL5). Depletion of FSTL5 in XPO1 inhibitor-sensitive cell lines rendered them resistant to the compound and induced the stabilization of Yes-associated protein (YAP), a transcriptional co-activator with a known role in conferring resistance to activation of mutant KRAS in pancreatic and lung cancer. Overexpression of YAP conferred resistance to XPO1 inhibitors, and inhibition of YAP conferred sensitivity.

These results suggest that alterations in the subcellular localization of certain proteins, particularly I $\mathrm{K} B a$ and YAP, could contribute to KRASmutant cancer growth. Therefore, components of the nuclear export machinery could be promising targets for the treatment of KRASmutant cancers.

Megan Cully

ORIGINAL ARTICLE Kim, J. et al. XPO1dependent nuclear export is a druggable vulnerability in KRAS-mutant lung cancer. Nature http://dx.doi.org/10.1038/nature19771 (2016) FURTHER READING Cox, A. D. et al. Drugging the undruggable RAS: Mission Possible? Nat. Rev. Drug Discov. 13, 828-851 (2014) 